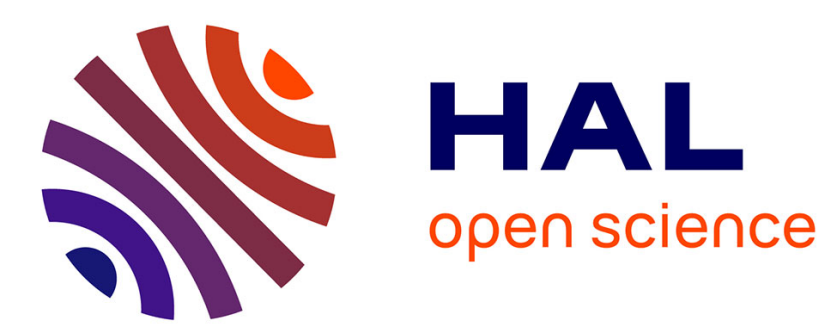

\title{
Pourquoi les immigrés portugais veulent-ils tant retourner au pays?
}

Manon Domingues dos Santos, François-Charles Wolff

\section{To cite this version:}

Manon Domingues dos Santos, François-Charles Wolff. Pourquoi les immigrés portugais veulent-ils tant retourner au pays?. 2010. hal-00449630

\section{HAL Id: hal-00449630 \\ https://hal.science/hal-00449630}

Preprint submitted on 22 Jan 2010

HAL is a multi-disciplinary open access archive for the deposit and dissemination of scientific research documents, whether they are published or not. The documents may come from teaching and research institutions in France or abroad, or from public or private research centers.
L'archive ouverte pluridisciplinaire HAL, est destinée au dépôt et à la diffusion de documents scientifiques de niveau recherche, publiés ou non, émanant des établissements d'enseignement et de recherche français ou étrangers, des laboratoires publics ou privés. 


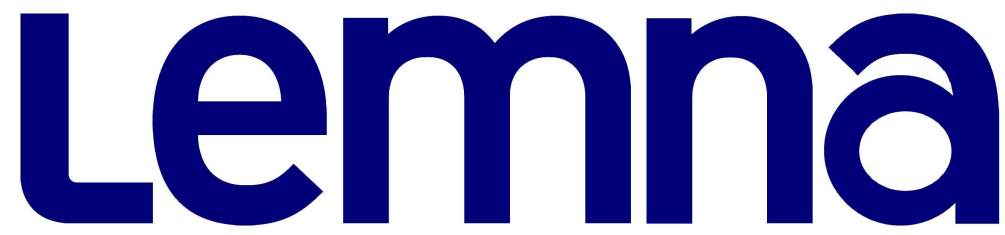

EA 4272

\title{
Pourquoi les immigrés portugais veulent-ils tant retourner au pays?
}

\author{
Manon Domingues Dos Santos* \\ François-Charles Wolff**
}

$2010 / 05$

${ }^{*}$ Correspondance. OEP - Université Paris-Est Marne-la-Vallée et CREST - INSEE

** LEMNA - Université de Nantes et CNAV et INED - Paris

Laboratoire d'Economie et de Management Nantes-Atlantique Université de Nantes

Chemin de la Censive du Tertre - BP 52231 44322 Nantes cedex 3 - France

www.univ-nantes.frliemn-iae/recherche

Tél. +33 (0)2 40141717 - Fax +33 (0)2 40141749 


\title{
Pourquoi les immigrés portugais veulent-ils tant retourner au pays ? ${ }^{\#}$
}

\author{
Manon Domingues Dos Santos* François-Charles Wolff**
}

Janvier 2010

Résumé: A partir des données de l'enquête sur le Passage à la Retraite des Immigrés réalisée en 2003, cet article cherche à comprendre pourquoi les ressortissants d'origine portugaise résidant en France se singularisent par des intentions de retour au pays à la retraite deux fois supérieures à celles de leurs homologues espagnols et italiens. Les différences communautaires relatives au niveau d'éducation, à la durée de séjour, à la maîtrise de la langue française, à la localisation des membres de la famille et à l'origine de l'éventuel conjoint expliquent une part importante de l'écart observé. L'application de techniques de décomposition non-linéaire révèle que près de $70 \%$ des écarts dans les intentions de retour entre les ressortissants du Portugal et les ressortissants d'Espagne et d'Italie s'expliquent par des différences entre les caractéristiques retenues, tandis qu'un tiers de l'écart observé est lié à l'effet différencié des facteurs influençant le souhait de localisation à la retraite.

Mots-clés: Migration, intentions de retour

Classification JEL: O15

\footnotetext{
${ }^{\#}$ Nous tenons à remercier deux rapporteurs anonymes de la revue pour leurs précieuses remarques et suggestions sur des versions préliminaires de ce texte ainsi que les participants aux Journées de Microéconomie Appliquée (Université de Saint-Denis, La Réunion).

* Correspondance. OEP, Université Paris-Est Marne-la-Vallée et CREST, INSEE, France.

Email : manondds@ensae.fr

${ }^{* *}$ LEMNA, Université de Nantes ; CNAV et INED, Paris, France.

Email: francois.wolff@univ-nantes.fr http://www.sc-eco.univ-nantes.fr/ fcwolff
} 


\section{Introduction}

Plus de la moitié des immigrés portugais qui vivent en France et qui sont âgés de 45 à 70 ans projettent de retourner dans leur pays natal une fois à la retraite. Curieusement, cette proportion est de moins d'un quart parmi leurs homologues espagnols et italiens. Pourquoi les intentions de retour des immigrés portugais sont-elles si singulières, alors que ces groupes d'immigrants sont si proches géographiquement? Dans la présente contribution, nous cherchons à apporter des éléments de réponse à cette question.

Analyser pourquoi les immigrés portugais se caractérisent par une si forte propension au retour est essentiel aussi bien pour appréhender les conséquences de leur migration sur l'économie d'accueil que sur les économies d'origine. En effet, au regard du pays d'accueil, la littérature théorique et empirique montre que les immigrés qui souhaitent retourner au pays sont susceptibles d'afficher une propension à l'emploi et à l'épargne plus importante, d'accepter des rémunérations plus faibles, ou d'être moins enclins à acquérir certaines compétences spécifiques au pays hôte telles que la maîtrise de la langue (Galor et Stark, 1990, 1991, Dustmann, 1997, 1999).

En ce qui concerne le pays d'origine, les émigrés qui veulent rentrer au pays y transfèrent une part plus importante de leurs ressources, ils y investissent à leur retour une partie de l'épargne qu'ils ont accumulée dans leur pays hôte, et ils y diffusent les compétences qu'ils ont acquises à l'étranger (Glytsos, 1997, Ahlburg et Brown, 1998, Dustmann et Kirschkamp, 2002, Mesnard, 2004, Domingues Dos Santos et Postel-Vinay, 2003, 2005). Ainsi, le retour des migrants conditionne largement leur comportement économique et, de là, l'incidence des flux migratoires sur les économies concernées. Il s'avère donc essentiel de comprendre les déterminants du retour des migrants (ou tout du moins de leurs intentions de retour) en étudiant le rôle des caractéristiques individuelles sur ces comportements, cet éclairage permettant au final de mieux appréhender les conséquences économiques des migrations.

En dépit de l'importance des enjeux liés aux retours des migrants, force est de constater que les contributions qui se sont intéressées aux déterminant du retour et aux caractéristiques des migrants susceptibles d'influencer cette décision demeurent peu nombreuses à ce jour. Il semble pourtant évident que l'impact économique du retour des migrants est conditionnel à leur âge, à leur qualification relative ou bien encore à leur situation familiale, et il convient de mieux connaître l'influence des caractéristiques individuelles sur la propension au retour. Le manque de travaux empiriques sur ce thème 
provient sans nul doute de l'absence de données appropriées, qui autorisent à mettre en relation la durée de séjour des immigrés dans le pays d'accueils et leurs caractéristiques ${ }^{1}$.

L’enquête intitulée Passage à la Retraite des Immigrés, réalisée en France en 2003, permet d'apporter un éclairage nouveau sur les intentions de retour des ressortissants étrangers. Les données recensées dans cette enquête sont exhaustives : elles portent sur la situation démographique, économique et sociale des enquêtés, et elles indiquent également leurs projets de retour. Il devient alors aisé d'étudier l'influence des caractéristiques individuelles sur les intentions de retour des migrants. Les données nous permettent surtout d'analyser de façon détaillée dans quelle mesure les caractéristiques observées expliquent pourquoi les migrants portugais affichent une propension au retour plus forte que celles de leurs homologues étrangers (mesurée à travers leurs intentions).

Dans la mesure où les contraintes migratoires institutionnelles diffèrent en fonction de l'origine des immigrés, nous avons choisi de confronter les intentions de retour des immigrés portugais à celles d'immigrés soumis à des contraintes migratoires institutionnelles similaires, à savoir les immigrés espagnols et italiens. En vertu des attributs de la citoyenneté européenne, les ressortissants de ces pays peuvent en effet librement circuler au sein de l'Union Européenne. Les travailleurs immigrés d'un pays membre bénéficient en outre des mêmes droits sociaux que les autochtones, et ils peuvent prétendre aux transferts publics résultant de leurs acquis en cas de retour au pays.

Contrairement notamment aux immigrés originaires d'Afrique, le retour des immigrés d'Europe du sud n'est pas contraint par la durée de leur titre de séjour, et leur éventuel retour au pays ne les empêche pas le cas échéant de réviser leurs choix ultérieurement ou de faire valoir leurs droits à la retraite. Autrement dit, nous comparons les intentions de retour au pays de communautés immigrées qui sont largement représentées en France et pour lesquelles l'éventuel retour n'est a priori pas affecté par des contraintes de nature institutionnelle.

Afin d'étudier la propension au retour des immigrés d'Europe du sud et de savoir s'il existe ou non d'éventuelle spécificités communautaires, nous procédons de la façon suivante. Dans une première étape, nous supposons que l'impact des variables explicatives retenues sur les intentions de retour des immigrés est indépendant de leur pays d'origine. Nous montrons que si le genre, l'âge ou la situation maritale n'influencent guère la propension au retour, les variables mesurant le capital humain de l'intéressé dans le contexte de son économie

\footnotetext{
${ }^{1}$ Il convient bien sûr de souligner la difficulté de disposer des données appropriées. Idéalement, il faudrait pouvoir suivre la trajectoire des immigrés lorsqu'ils arrivent dans le pays d'accueil et retournent ensuite au pays.
} 
d'accueil, en particulier le nombre d'années d'éducation, la durée de séjour ou la maîtrise de la langue française, ont un impact important. Toutefois, l'effet de l'origine reste significatif une fois que l'on prend en considération les caractéristiques des immigrés : la propension au retour des portugais est supérieure de près de 14 points de pourcentage à celle de leurs homologues espagnols et italiens. Les intentions de retour des immigrés apparaissent également fortement corrélées aux attaches que les immigrés entretiennent à la fois avec les pays d'origine et d'accueil, ces liens étant mesurés à travers la localisation des membres de leur cellule familiale et l'origine de leur conjoint.

Dans une seconde étape, nous cherchons à apprécier la part des spécificités communautaires qui relève de différences ayant trait aux caractéristiques observables des immigrés et celle qui s'explique par un impact différent de ces caractéristiques sur les intentions de retour. Pour répondre à cette question, nous appliquons des techniques de décomposition adaptées à des variables dépendantes non-linéaires, proposées récemment par Yun (2004). Nos résultats révèlent que les différences dans les intentions de retour entre les Portugais et les Espagnols et Italiens sont dans une proportion non négligeable (30\%) liées à des différences dans l'impact des facteurs explicatifs retenus. Il existe donc de réelles spécificités communautaires au regard des intentions de retour.

La suite de cette contribution est organisée de la façon suivante. Dans la section 2, nous présentons les enseignements de la littérature théorique et empirique sur les déterminants de la propension au retour. La section 3 présente les principales statistiques descriptives relatives à l'échantillon retenu. La section 4 analyse les déterminants de la propension au retour en supposant que leur impact n'est pas conditionné par l'origine des intéressés. La section 5 relâche cette hypothèse en proposant notamment une décomposition de type Oaxaca-Blinder adaptée au cas discret. Finalement, la section 6 conclut.

\section{Les déterminants du retour des migrants}

Comme le souligne Dustmann (1996), les migrations avec retour constituent plus la règle que l'exception. A titre d'exemple, Bohning (1981) estime que plus des deux tiers des immigrés admis en RDA entre 1961 et 1976 sont retournés ensuite dans leur pays d'origine. D'après Jasso et Rosenzweig (1982), entre $20 \%$ et $50 \%$ des immigrés américains (suivant leurs nationalités d'origine) en ont fait de même pendant les années 70. Les législations migratoires des pays d'accueil expliquent bien sûr le caractère temporaire de 
certaines migrations pour ceux qui ne disposent que d'un titre de séjour temporaire. Les orientations actuelles des politiques migratoires appliquées dans les pays d'accueil traditionnels laissent augurer une intensification de ce motif (Domingues Dos Santos, 2006).

Toutefois, une part substantielle des migrations avec retour ne résulte pas de contraintes institutionnelles. C'est notamment le cas des migrations entre les pays de l'Union Européenne des ressortissants des pays membres, argument motivant notre choix de limiter notre étude aux cas des immigrés français originaires d'Europe du sud. La littérature théorique analysant les déterminants du retour migratoire identifie plusieurs motifs rationnels et non exclusifs de retour.

En univers incertain, la migration initiale peut tout d'abord s'être effectuée sur la base d'anticipations erronées. Ainsi, dans l'éventualité où le migrant aurait a priori surestimé l'avantage à migrer, il peut une fois l'erreur constatée choisir rationnellement de retourner dans son pays d'origine ou de migrer vers une autre destination (Borjas et Brastberg, 1996). Les immigrés originaires d'Europe du sud étant soumis aux mêmes contraintes migratoires institutionnelles depuis 1986, date de l'entrée dans l'Union Européenne de l'Espagne et du Portugal, nous pouvons supposer que le retour occasionné par une migration spéculative n'a pas a priori induit de biais de sélection entre les communautés étudiées. De même, tout immigré d'Europe du sud qui décide de rentrer au pays peut le cas échéant réviser son choix et retourner en France.

En univers certain, en présence de préférences pour la consommation dans le pays d'origine, les migrants peuvent choisir de rentrer après avoir accumulé une épargne conséquente dans le pays hôte (Djajic et Milbourne, 1988). Pour Djajic (1989), le retour peut être rationnel quand le pouvoir d'achat des avoirs capitalisés dans l'économie d'accueil est plus élevé dans l'économie d'origine. Le retour est également motivé lorsque les rendements des compétences acquises à l'étranger sont plus élevés dans le pays d'origine. Stark (1992) montre que le fait de prendre en compte les interactions sociales peut également expliquer le retour lorsque l'émigration a permis d'améliorer suffisamment le statut relatif du migrant dans son pays d'origine.

Une dernière explication, suggérée plus récemment par Dustmann (2003), met en jeu l'altruisme familial. Si les parents se soucient du bien-être de leurs enfants au sens beckerien, ils prennent également en compte l'incidence de leurs propres choix de localisation sur la situation future de leur progéniture. L'effet net de l'altruisme sur la probabilité de retour est dans ce cas indéterminé. Si les enfants peuvent bénéficier d'un meilleur niveau de vie en restant en France, les parents privilégieront d'y rester. A l'inverse, ils opteront pour le retour 
au pays s'ils estiment que leurs enfants y trouveront un environnement économique et culturel plus favorable ${ }^{2}$. Ainsi, une part des écarts relatifs à la propension au retour peuvent s'expliquer par des différences socio-économiques entre les pays de retour, notamment en termes de pouvoir d'achat, de services publics ou de rendements des actifs capitalisés.

Au regard de la sélectivité du processus de retour, les travaux antérieurs mettent essentiellement en évidence le rôle de la qualification, de l'âge et de l'intensité des attaches du migrant avec les pays d'accueil et d'origine. En ce qui concerne la qualification, Ramos (1992) et Rooth et Saarela (2007) montrent que le retour des migrants accentue la sélection qui caractérise les flux migratoires initiaux. Lorsque les émigrés sont initialement relativement peu qualifiés, ce sont les plus qualifiés d'entre eux qui retournent au pays tandis que lorsque qu'ils sont ex ante relativement qualifiés, ce sont les moins qualifiés qui rentrent.

Steiner et Velling (1994) ainsi que Constant et Massey (2003) mettent en évidence une plus forte propension au retour à l'âge de la retraite. La propension au retour diminue également avec le niveau d'intégration du migrant dans son pays d'accueil, alors qu'elle augmente avec l'intensité de ses liens avec son pays d'origine ${ }^{3}$. Ainsi, la propension augmente avec l'âge d'arrivé et diminue avec la durée de séjour, la maîtrise de la langue du pays d'accueil, la naturalisation, la propriété d'un logement ou la présence d'enfants et du conjoint. En revanche, la propension au retour augmente lorsque les migrants transfèrent des revenus vers leur pays d'origine, y ont des enfants en bas âge, et lorsque leur conjoint ont leur conjoint là-bas.

Néanmoins, certaines caractéristiques telles que la maîtrise de la langue du pays d'accueil, l'ampleur des transferts ou la localisation des enfants peuvent elles même être influencées par l'aspiration au retour. A ce titre, De Coulon et Wolff (2006) montrent que la localisation des enfants, du conjoint et du migrant sont en partie co-déterminées. Ainsi, toute corrélation éventuellement mise en évidence entre la propension au retour et le degré d'intégration du migrant ou bien entre la propension au retour et les liens avec le pays d'origine est a priori sujette à un biais de simultanéité qui en empêche une interprétation causale.

\footnotetext{
${ }^{2}$ Dans la mesure où les parents valorisent plutôt les carrières de leurs fils et des éléments liés aux traditions ou au mariage jouent davantage pour les filles, les effets de la composition par sexe de la fratrie vont influencer les décisions de retour des parents (Dustmann, 2003).

${ }^{3}$ Voir en outre Dustmann (1993, 2003), Duleep (1994), Steiner et Velling (1994), Reagan et Olsen (2000), Constant et Massey (2003) et De Coulon et Wolff (2006).
} 


\section{Les données}

Pour étudier les intentions de retour des migrants vivant en France, nous utilisons les données de l'enquête Passage à la Retraite des Immigrés (PRI), réalisée par la CNAV et l'INSEE en 2003. Elle porte sur la population immigrée âgée de 45 à 70 ans, qui réside en France à la date de l'enquête, la population d'origine étrangère étant définie par le lieu de naissance (hors de France) et la nationalité de naissance (non française). Ceci n'exclut donc pas les immigrés aujourd'hui Français par acquisition de la nationalité, mais exclut les Français qui sont nés à l'étranger.

Cette enquête obtenue par un sondage aléatoire à partir du recensement de la population est représentative de la population immigrée âgée vivant en France, et l'échantillon initial comprend 6211 personnes. Elle contient les informations usuelles relatives aux caractéristiques démographiques et socio-économiques de l'enquêté telles que son âge, sa situation familiale ou encore son éducation. Elle recense également des informations originales concernant son histoire migratoire, en particulier sa date d'arrivée ou son niveau de maîtrise de la langue française. Enfin, l'enquête précise aussi les projets de retraite des migrants, pour celles et ceux qui ne l'ont pas encore prise, et sur leur choix de vie après la retraite.

Afin d'analyser la propension au retour des immigrés, nous utilisons une question portant sur l'intention de retour exprimée par l'enquêté. Recourir à des questions relatives aux intentions et non aux réalisations est usuel dans la littérature sur les migrations. Dustmann (2003) a recours à cette stratégie sur des données longitudinales allemandes et montre que les projets de retour déclarés se concrétisent très fréquemment. Concernant les intentions de retour des immigrés, l'enquête PRI distingue plusieurs choix de localisation à la retraite. Le migrant peut soit rester en France, soit retourner au pays d'origine, soit pratiquer le va-etvient entre la France et son pays d'origine, ou bien encore ne pas avoir d'idée précise sur son intention ${ }^{4}$. Nous construisons donc une variable discrète qui prend pour valeur 1 lorsque l'enquêté émet l'intention de pas rester en France à la retraite et 0 dans le cas contraire 5 .

Comme nous l'avons expliqué, nous centrons notre étude sur les ressortissants originaires d'Espagne, d'Italie, et du Portugal. Par définition, nous limitons également notre étude aux immigrés qui ne sont pas encore partis à la retraite à la date de l'enquête.

\footnotetext{
${ }^{4}$ Plus précisémment, la question suivant est posée aux enquêtés. « Au cours de votre retraite, souhaitez-vous :

(1) Demeurer où vous êtes (en France) - (2) Aller ailleurs en France - (3) Retourner dans votre pays d'origine -

(4) Aller vivre dans un autre pays - (5) Passer du temps en France et dans votre pays d'origine - (6) Passer du temps en France et dans un autre pays - (7) Ne sais pas ou ne désire pas répondre »

${ }^{5}$ Les choix de va-et-vient ont été analysés par Attias-Donfut et Wolff (2005) et de Coulon et Wolff (2006).
} 
L'échantillon retenu comprend au total 1479 enquêtés, dont 712 sont d'origine espagnole ou italienne et 767 d'origine portugaise.

Globalement, l'intention de retour est formulée par plus de $37 \%$ des ressortissants d'Europe du Sud. Toutefois, comme le montre le Tableau 1, il existe de fortes différences entre les groupes. Alors que le retour est projeté par 13\% des immigrés italiens, la propension au retour de leurs homologues espagnols avoisine les 25\%. Dans le même temps, près de la moitié des immigrés portugais anticipent de retourner au pays. Il existe donc des différences très fortes dans les intentions de retour à la retraite entre les immigrés espagnols et italiens et les immigrés portugais.

\section{Insérer Tableau 1}

Les caractéristiques relatives au sexe, à l'âge et à la situation familiale des enquêtés apparaissent similaires pour les deux groupes. En revanche, les deux communautés se distinguent au regard de leur localisation géographique. Les immigrés portugais sont très fortement concentrés en région parisienne, tandis que la présence d'immigrés espagnols et italiens s'avère plus marquée dans le sud-est de la France. Nous observons également des différences substantielles au regard du niveau d'éducation et de rémunération. Les ressortissants d'origine portugaise ont réalisé des études sensiblement plus courtes, de près de 3 ans en moyenne. Ils perçoivent aussi des revenus moindres, même si l'écart observé demeure faible (3.5\% en moyenne).

Les trajectoires migratoires diffèrent également entre les deux communautés. L'immigration italienne et espagnole apparaît plus ancienne que l'immigration portugaise. Alors que plus de la moitié des italiens ont immigré avant les années 60, plus de la moitié des espagnols sont arrivés en France entre 1960 et 1970. L'immigration portugaise s'avère plus tardive et massive, puisque plus des deux tiers des ressortissants portugais ont migré entre 1965 et 1975. Par ailleurs, les migrants portugais affichent un âge à la migration plus élevé. Ils ont en moyenne immigré à 21 ans, alors que l'âge d'arrivée en France n'est que de 14 ans pour leurs homologues espagnols et italiens (près d'un tiers des italiens ont immigré dans leur plus jeune âge). Les deux communautés ayant sensiblement le même âge moyen, la durée de migration est donc relativement plus longue pour les migrants espagnols et italiens $(39,8$ ans au lieu de 32,1 ans).

L'immigration portugaise présente donc certaines caractéristiques remarquables. Les immigrés portugais sont arrivés plus tardivement et massivement en France, à un âge plus avancé, et ils affichent donc une durée moyenne de séjour plus faible. Ces caractéristiques contribuent très certainement à expliquer les plus grandes difficultés des ressortissants du 
Portugal à parler parfaitement le français $(43,3 \%$ au lieu de $20,6 \%$ pour les Espagnols et Italiens).

Les deux communautés se distinguent par ailleurs par les liens familiaux qu'elles entretiennent avec leur pays d'origine et d'accueil. Si près de $90 \%$ des immigrés des deux groupes ont au moins un enfant vivant en France et près de $10 \%$ ont au moins un enfant vivant au pays, les deux communautés se singularisent avant tout au regard de la localisation de leurs parents, de leurs frères et sœurs et de l'origine de leur conjoint. Si $40 \%$ des ressortissants d'origine portugaise ont au moins un parent vivant au pays, cette proportion n'est que de $23 \%$ pour les ressortissants espagnols et italiens. De manière similaire, une proportion plus importante de portugais ont au moins un membre de leur fratrie au pays ${ }^{6}$. La moitié des ressortissants d'origine espagnole ou italienne a un conjoint né en France. A l'inverse, cette proportion n'excède pas $14 \%$ pour les ressortissants d'origine portugaise.

Ces disparités communautaires au regard de la localisation des membres de la famille s'expliquent en partie par les différences d'âge à la migration. Les immigrés portugais étant arrivés en France à 21 ans en moyenne, ils sont plus enclins que leurs homologues espagnols ou italiens (arrivés à 14 ans en moyenne) à avoir migré sans leurs parents ou à avoir eu un conjoint avant la migration. Enfin, certaines caractéristiques peuvent a priori aussi bien expliquer que manifester un attachement relativement plus important des immigrés portugais à leur pays d'origine. Ainsi, les portugais sont beaucoup moins enclins à acquérir la nationalité française, ils sont moins souvent propriétaire de leur logement en France et ils envoient beaucoup plus fréquemment des fonds vers leur pays d'origine.

$\mathrm{Au}$ final, ces premiers résultats descriptifs mettent en évidence d'un côté des écarts substantiels entre les intentions de retour parmi les migrants originaires d'Europe du Sud, de l'autre des écarts substantiels dans les caractéristiques individuelles susceptibles d'influencer ces intentions. Nous analysons à présent dans quelle mesure les différences dans les caractéristiques individuelles observées expliquent pourquoi les ressortissants du Portugal expriment davantage le souhait de retour au pays à la retraite que leurs homologues espagnols et italiens.

\footnotetext{
${ }^{6}$ En revanche, il n'existe aucune différence entre les deux populations en ce qui concerne la présence d'autres membres de la famille au pays, mais l'enquête n'apporte aucune précision sur la nature des liens familiaux avec ces autres membres.
} 


\section{Les déterminants des intentions de retour}

Compte tenu de la nature discrète de la variable à expliquer, nous estimons la probabilité pour un migrant de retourner au pays d'origine par un modèle Probit. Il existe donc une variable latente associée au souhait de retourner dans son pays d'origine à la retraite, cette variable étant positive lorsque le migrant exprime l'intention de rentrer (et négative ou nulle dans le cas contraire). La régression est estimée sur l'échantillon global des 1479 migrants. L'introduction d'une variable muette égale à l'unité lorsque le migrant est d'origine portugaise permet d'évaluer les éventuelles différences suivant l'origine une fois prise en compte l'incidence des variables explicatives.

En estimant une régression unique, nous supposons toutefois ici que les effets des facteurs explicatifs sur les intentions de retour ne sont pas conditionnés par le pays d'origine des migrants. La pertinence de cette hypothèse est étudiée dans la section suivante. Les résultats des estimations sont reportés dans le tableau 2. Dans une première étape, nous introduisons uniquement les variables démographiques et socio-économiques usuelles relatives au capital humain et au revenu des immigrés (Colonnes 1 et 2, Tableau 2). Deux principaux résultats sont mis en évidence.

\section{Insérer Tableau 2}

Tout d'abord, le genre, l'âge et la situation maritale n'influencent pas vraiment la propension au retour. A l'inverse, les variables qui mesurent le capital humain de l'intéressé dans le contexte de son économie d'accueil ont un impact substantiel : l'intention de retour diminue avec le nombre d'années d'éducation, mais elle augmente avec le revenu de l'intéressé. Ce dernier effet s'explique vraisemblablement par la définition de la variable dépendante, qui inclut de nombreuses situations de va-et-vient. Partager son temps entre deux pays impliquant des coûts de transport et de logement importants, et ceux-ci peuvent être plus facilement pris en charge par les ménages aisés.

Par ailleurs, les variables caractérisant le niveau d'intégration des immigrés, telles que leur durée de séjour ou leur maîtrise de la langue française, ont un impact négatif sur leur propension au retour, l'incidence de cette seconde variable étant très élevée. Il convient toutefois de noter, comme le souligne Dustmann (1999), que l'intention de retour et l'investissement en capital humain spécifique au pays d'accueil, dont le niveau de maitrise de la langue est une composante, peuvent être conjointement déterminés par le migrant.

L'impact de l'origine reste également prépondérant après contrôle des caractéristiques individuelles. Si la probabilité de retour estimée à partir des caractéristiques moyennes de 
l'échantillon est de $34 \%$, cette probabilité augmente de 24 points de pourcentage lorsque l'enquêté est d'origine portugaise. Les plus faibles niveaux relatifs de durée de séjour et de maîtrise de la langue française contribuent à expliquer de façon substantielle la plus forte propension au retour des portugais. Si celle-ci diminue de près de 10 points de pourcentage lorsque ces variables sont prises en considération dans la régression, la propension au retour des immigrés d'origine portugaise demeure néanmoins supérieure de près de 14 points de pourcentage à celle de leurs homologues espagnols et italiens.

Après avoir considéré l'impact des caractéristiques démographiques et socioéconomiques sur la propension au retour, nous introduisons dans une seconde étape un ensemble de variables mesurant les attaches que les immigrés entretiennent avec leur pays d'accueil et leur pays d'origine (Colonne 3, Tableau 2). L'interprétation causale des corrélations observées est toutefois sujette à caution. En effet, comme nous l'avons déjà souligné, certaines caractéristiques telles que l'ampleur des transferts réalisés par les migrants vers leur pays d'origine, la localisation de leurs enfants, l'achat d'une résidence dans leur pays d'accueil ou leur naturalisation française peuvent être fortement influencées par l'aspiration au retour.

La localisation d'un enfant ou d'un parent au pays est fortement corrélée avec le projet de retour. Maintenir des contacts fréquents avec ses ascendants et descendants directs est en effet plus facile lorsque ceux-ci vivent à proximité ${ }^{7}$. Outre la localisation des proches, le lieu de naissance du conjoint apparaît également comme un élément clef. Avoir un conjoint né en France réduit en effet très fortement la propension au retour. Se sentir victime de discrimination accroît au contraire de façon significative le projet de retour. Enfin, l'acquisition de la nationalité française, être propriétaire de son logement en France ou renvoyer de l'argent dans le pays d'origine ont également un impact substantiel avec des signes conformes aux attentes.

Prendre en considération les attaches que les immigrés entretiennent avec leurs pays d'accueil et d'origine réduit de près de 8 points de pourcentage le différentiel communautaire résiduel. L'impact marginal de l'origine portugaise sur la probabilité de retour n'est plus que de 6 points de pourcentage avec cette nouvelle spécification. Clairement, si l'impact propre à l'origine diminue lorsque l'on intègre ces liens, il demeure toutefois non-négligeable.

\footnotetext{
${ }^{7}$ De nombreuses études sur les relations familiales ont montré que les contacts et visites étaient beaucoup plus importants lorsque les parents et les enfants vivent à proximité les uns des autres (Jellal et Wolff, 2002, Sloan et alii, 2003).
} 
Pour résumer, la prise en considération de caractéristiques ayant trait à la situation démographique ou socio-économique ainsi qu'aux attaches que les immigrés entretiennent avec leur pays d'accueil et d'origine permet d'expliquer largement la plus forte propension au retour des immigrés portugais. Toutefois, une partie substantielle de l'incidence de l'origine portugaise sur la propension au retour demeure inexpliquée par les variables prises en compte dans les régressions.

\section{Une décomposition des écarts observés}

Les différences de propension au retour entre communautés peuvent être attribuables soit à des différences portant sur le niveau des caractéristiques observables des deux communautés, soit à un impact différent de ces caractéristiques sur la propension au retour de leurs membres ${ }^{8}$. Pour répondre à cette interrogation, nous utilisons des techniques de décomposition de type Oaxaca-Blinder qui permettent d'évaluer le poids respectif des différences relatives aux caractéristiques observées et celles relatives à leur rendement 9 . Toutefois, la variable d'intérêt n'étant pas continue, nous retenons une méthode de décomposition adaptée au cas des variables binaires.

Plus précisément, le principe d'une décomposition de type Oaxaca-Blinder est le suivant. Supposons que l'on cherche à expliquer les différences observées pour une variable d'intérêt continue $Y$ entre les deux groupes constitués respectivement par les portugais d'un côté (groupe $A$ ) et par les italiens et espagnols de l'autre (groupe $B$ ). Soient $X_{i}$ un vecteur de variables explicatives et $\beta_{i}$ le vecteur de coefficients associés, avec $i=A, B$. La différence totale $Y_{A}-Y_{B}=X_{A} \beta_{A}-X_{B} \beta_{B}$ peut s'écrire sous la forme suivante:

$$
Y_{A}-Y_{B}=\left(X_{A}-X_{B}\right) \beta_{A}+X_{B}\left(\beta_{A}-\beta_{B}\right)
$$

On construit donc une équation auxiliaire prenant en compte les caractéristiques observables d'un groupe, en l'occurrence le groupe $B$ dans l'exemple précédent, et les coefficients estimés obtenus par régression linéaire sur le groupe $A$. Le premier terme $\left(X_{A}-X_{B}\right) \beta_{A}$ indique les écarts entre les deux groupes qui sont liés aux différences de caractéristiques entre les deux groupes, tandis que le second terme $X_{B}\left(\beta_{A}-\beta_{B}\right)$ mesure les écarts liés aux différences de coefficients pour les caractéristiques retenues dans la

\footnotetext{
${ }^{8}$ Les écarts peuvent aussi être attribuables à des caractéristiques non observées, ou à des différences dans l'impact de celles-ci.

${ }^{9}$ Ces techniques de decomposition ont été originellement utilisées pour comprendre les écarts de revenus salariaux par sexe. Pour un apercu de la literature, se reporter à Oaxaca et Ransom (1998).
} 
régression ${ }^{10}$. Cette décomposition peut être menée à la fois au niveau agrégé et pour chaque variable explicative prise en compte dans l'analyse.

Il importe de noter que plusieurs décompositions peuvent être menées pour expliquer la différence $Y_{A}-Y_{B}$ : il existe généralement une dépendance de sentier dans l'application des méthodes de Oaxaca-Blinder. Dans le cas général, il vient (Oaxaca et Ransom, 1994) :

$$
Y_{A}-Y_{B}=\left(X_{A}-X_{B}\right) \beta^{*}+X_{A}\left(\beta_{A}-\beta^{*}\right)+X_{B}\left(\beta^{*}-\beta_{B}\right)
$$

où $\beta^{*}$ est définie comme étant la moyenne pondérée suivante :

$$
\beta^{*}=\Omega \beta_{A}+(I-\Omega) \beta_{B}
$$

Différentes décompositions sont donc possibles, suivant les valeurs prises par la matrice de pondération $\Omega$. Ainsi, lorsque $\Omega=I$, on retrouve la décomposition qui est donnée $\operatorname{par}(1)$. A l'inverse, si $\Omega=0$, alors $\beta^{*}=\beta_{B}$ et donc $Y_{A}-Y_{B}=\left(X_{A}-X_{B}\right) \beta_{B}+X_{A}\left(\beta_{A}-\beta_{B}\right)$ : le groupe factice qui est retenu pour la décomposition a les caractéristiques observées de la population $A$. D'autres alternatives consistent à retenir $\Omega=0,5$ (Reimers, 1983) ou bien $\beta^{*}=\beta=\left(X^{\prime} X\right)^{-1}\left(X^{\prime} Y\right)$ (Neumark, 1988). Dans ce dernier cas, les coefficients retenus sont obtenus à partir d'une régression estimée sur la population globale composée des deux groupes $A$ et $B$.

Si une telle décomposition est désormais usuelle lorsque la variable d'intérêt est continue, le problème est un peu plus complexe dans le cas d'une variable discrète. Cette question a néanmoins fait l'objet de développements récents, en particulier Even et Macpherson (1993), Nielsen (1998), Fairlie (1999, 2005), Yun (2004), ou bien Bauer et Sinning (2008). Tous ces auteurs proposent des généralisations de la décomposition BlinderOaxaca à des fonctions non-linéaires. Le problème principal vient du fait que l'espérance conditionnelle $E(Y \mid X)$ n'est plus nécessairement égale à $X \beta$, contrairement à un modèle linéaire où $E(Y \mid X)=X \beta$.

Considérons à présent le cas d'une variable binaire. Soit $P_{A}=\Phi\left(X_{A} \beta_{A}\right)$ la probabilité mesurant les intentions de retour pour le groupe $A$ (les portugais) et $P_{B}=\Phi\left(X_{B} \beta_{B}\right)$ la probabilité correspondante pour le groupe $B$ (les italiens et les espagnols), avec $\Phi($.$) la$ fonction de distribution de la loi normale univariée. Suivant Yun (2004), la différence dans les intentions moyennes de retour pour les groupes $A$ et $B$, notées $\bar{P}_{A}$ et $\bar{P}_{B}$, est donnée par:

\footnotetext{
${ }^{10} \mathrm{Ce}$ second terme est généralement appréhendé en termes de discrimination lorsque la décomposition porte sur des équations de salaires estimées respectivement pour les hommes et pour les femmes.
} 


$$
\bar{P}_{A}-\bar{P}_{B}=\left[\overline{\Phi\left(X_{A} \beta_{A}\right)}-\overline{\Phi\left(X_{B} \beta_{A}\right)}\right]+\left[\overline{\Phi\left(X_{B} \beta_{A}\right)}-\overline{\Phi\left(X_{B} \beta_{B}\right)}\right]
$$

Autrement dit, il suffit de calculer les probabilités moyennes de retour pour le groupe $A$, pour le groupe $B$, et pour un groupe fictif qui a ici les caractéristiques $X_{B}$ du groupe $B$ et le comportement du groupe $A$ donné par les coefficients $\beta_{A}$ (là encore, le groupe de référence pouvant être modifié). Il est également possible de mener une décomposition détaillée de la différence $\bar{P}_{A}-\bar{P}_{B}$. Celle-ci indique alors les contributions de chaque variable à la fois pour les écarts liés aux différences de caractéristiques observables et pour les écarts liés aux différences de coefficients. L'application d'un développement de Taylor à l'ordre un permet d'écrire (4) sous la forme suivante (Yun, 2004):

$$
\bar{P}_{A}-\bar{P}_{B}=\sum_{k=1}^{K} W_{\Delta X}^{k}\left[\overline{\Phi\left(X_{A} \beta_{A}\right)}-\overline{\Phi\left(X_{B} \beta_{A}\right)}\right]+\sum_{k=1}^{K} W_{\Delta \beta}^{k}\left[\overline{\Phi\left(X_{B} \beta_{A}\right)}-\overline{\Phi\left(X_{B} \beta_{B}\right)}\right]
$$

avec $K$ le nombre de variables explicatives inclues dans le vecteur $X_{i, i=A, B}$. Les poids $W_{\Delta X}^{k}$ et $W_{\Delta \beta}^{k}$ sont respectivement donnés par:

$$
\begin{gathered}
W_{\Delta X}^{k}=\frac{\left(\bar{X}_{A}^{k}-\bar{X}_{B}^{k}\right) \beta_{A}^{k}}{\left(\bar{X}_{A}-\bar{X}_{B}\right) \beta_{A}} \\
W_{\Delta X}^{k}=\frac{\bar{X}_{B}^{k}\left(\beta_{A}^{k}-\beta_{B}^{k}\right)}{\bar{X}_{B}\left(\beta_{A}-\beta_{B}\right)}
\end{gathered}
$$

avec $\sum W_{\Delta X}^{k}=1, \sum W_{\Delta \beta}^{k}=1$, et $\bar{X}_{i}^{k}$ est la valeur moyenne de la variable explicative $X^{k}$ pour le groupe $i(i=A, B)$. S'il est aisé de mettre en œuvre les calculs nécessaires pour la décomposition, il convient de noter que celle-ci ne reste qu'une approximation (Yun, 2004). Elle comporte en particulier deux erreurs d'approximation : la première est relative au recours à une expansion de Taylor, la seconde vient du fait que l'on considère la prédiction de la moyenne plutôt que la moyenne de la prédiction. Néanmoins, en dépit de cette limitation, Fairlie (2005) souligne qu'une telle décomposition discrète est bien plus appropriée que la méthode standard à la Oaxaca-Blinder en présence d'une variable dépendante binaire.

Les résultats obtenus sont reportés dans le Tableau 3. Les techniques de décomposition non linéaires permettent de préciser les poids relatifs des écarts dans les intentions de retour qui sont respectivement liés à des différences de caractéristiques entre les portugais et les espagnols et italiens et à des différences dans le rôle de ces facteurs explicatifs pour ces deux populations. Pour les différences de caractéristiques, nous avons également calculé les contributions respectives de chaque facteur explicatif retenu ainsi que les écartstype correspondant (obtenus par une technique de bootstrap). Enfin, nous avons mis en œuvre 
les décompositions pour différentes populations de référence, en particulier $\beta^{*}=\beta_{A}, \beta^{*}=\beta_{B}$ et $\beta^{*}=\beta$ (décomposition à la Neumark).

\section{Insérer Tableau 3}

Les résultats de la décomposition agrégée révèlent qu'environ $70 \%$ des écarts dans les intentions de retour entre les ressortissants du Portugal et les ressortissants d'Espagne et d'Italie s'expliquent par des différences dans les caractéristiques observables de ces deux groupes. Cette proportion varie en fonction de la population retenue, avec des valeurs allant de $63.7 \%$ pour $\beta^{*}=\beta_{A}$ à $74.1 \%$ pour $\beta^{*}=\beta^{11}$. Il en résulte que près de $30 \%$ de l'écart initial vient de différences dans les coefficients des régressions, avec une proportion allant de $25,9 \%$ à $36,3 \%$. Ainsi, un tiers de l'écart net observé sur les intentions de retour est lié à l'effet différencié cumulé des facteurs explicatifs retenus qui influencent le souhait de localisation à la retraite. En clair, si les deux communautés avaient en moyenne les mêmes caractéristiques, le différentiel de propension au retour diminuerait de près de $70 \%$.

Compte tenu de la difficulté d'interprétation de la proportion inexpliquée des écarts dans les intentions de retours (Fairlie, 2005), seules les contributions des variables relatives à la différence de caractéristiques entre les deux groupes sont indiquées dans le Tableau 3. Deux variables exercent alors un rôle prépondérant : il s'agit de la durée de la migration et de la localisation en région parisienne. Avec une décomposition à la Neumark, ces deux facteurs expliquent plus de $80 \%$ de l'incidence totale relative aux caractéristiques observables. Si les deux populations considérées avaient les mêmes durées de séjour en France, l'écart dans les intentions de retour serait réduit de 53,8\%. Enfin, le fait d'avoir des difficultés à parler le français et le nombre d'années d'éducation joue aussi un rôle significatif, même si les effets observés sont aussi plus sensibles à la dépendance de sentier.

\section{Discussion et conclusion}

Les migrations internationales ont de longue date été étudiées dans le contexte du marché du travail. Avec le vieillissement de la population immigrée, les économistes ne s'intéressent aujourd'hui plus seulement aux performances sur le marché du travail des ressortissants étrangers. Ils cherchent également à analyser la migration sur l'ensemble du cycle de vie et en plaçant celle-ci dans sa dimension intergénérationnelle. A partir de l'exploitation de l'enquête PRI, nous avons précisé dans cette étude les intentions de retour à

\footnotetext{
${ }^{11}$ L'incertitude sur le choix de la population de référence peut être vue comme une source d'imprécision supplémentaire dans la décomposition.
} 
la retraite des ressortissants originaires d'Europe du Sud qui vivaient en France en 2003. Nos principaux résultats sont les suivants.

Tout d'abord, il existe un véritable ancrage de la population originaire d'Europe du Sud vivant en France. En effet, la proportion de migrants souhaitant retourner définitivement au pays demeure limitée. Ensuite, nous montrons que les caractéristiques individuelles démographiques et économiques ainsi que les attaches que les immigrés entretiennent tant avec leur pays d'origine qu'avec leur pays d'accueil ont une influence conséquente sur leur souhait de retour au pays. La temporalité des migrations est donc largement conditionnée par la structure des flux migratoires initiaux dont le niveau d'éducation, la durée de séjour, la maîtrise de la langue, la localisation des membres de la cellule familiale ou bien encore l'origine du migrant sont des éléments essentiels.

Enfin, nous soulignons les spécificités communautaires de la propension au retour entre les immigrés d'origine portugaise et leurs homologues italiens et espagnols. Les ressortissants portugais exprimant plus fréquemment un désir de retour au pays, nous avons analysé dans quelle mesure cette spécificité s'expliquait par des différences relatives aux caractéristiques observables des migrants ou par des effets différenciés de ces variables explicatives sur l'intention de retour. L'application de techniques de décomposition adaptées au cas non-linéaire révèle que si près de $70 \%$ de l'écart total observé entre les deux groupes de migrants provient des différences dans les caractéristiques retenues, l'influence de ces caractéristiques n'est toutefois pas homogène entre les communautés. Il existe donc bien des spécificités communautaires dans le comportement de retour des immigrés.

Si les caractéristiques individuelles retenues dans notre analyse n'expliquent qu'en partie la plus forte propension au retour des migrants portugais, il convient de s'interroger sur les facteurs susceptibles d'expliquer le résidu. A ce stade, il est intéressant de souligner qu'une part non négligeable des projets de retour au pays semble antérieure à la migration et que les intentions de retour ont tendance à se maintenir tout au long du séjour. Les données fournissent à ce sujet une information d'intérêt, puisque l'enquête comprend une question sur les projets initiaux à l'époque où les migrants sont arrivés en France. Certes, les résultats ne sont sans doute pas sans biais de justification, car il est tout à fait possible que les enquêtés répondent à la question en référence à leur intention actuelle plutôt qu'à leur intention originelle (sans aucun doute délicate à se remémorer).

De nouveau, des différences significatives sont mises en évidence à travers l'enquête PRI entre les deux groupes de migrants. D'après le Tableau 4, si plus d'un tiers des migrants d'origine espagnole ou italienne avaient pour projet initial de demeurer définitivement en 
France, cette proportion n'était que d'un cinquième parmi les portugais. Parallèlement, plus de la moitié de ces derniers avaient pour intention première de rentrer au pays, contre seulement un tiers de leurs homologues espagnols et italiens. Les différences par pays d'origine dans les comportements de retour en fin de carrière tendent donc à se maintenir tout au long de la vie active.

\section{Insérer Tableau 4}

Pour finir, il convient de souligner les deux principales limites de cette contribution, qui constituent autant de voies de recherche à explorer. En premier lieu, les données disponibles se limitent aux intentions de retour des immigrés âgés de plus de 45 ans et présents en France. Nous ne pouvons donc ni apprécier dans quelle mesure ces souhaits seront ou non concrétisés, ni étudier le comportement de retour au cours d'une majeure partie de la vie active. En ce sens, il est vraisemblable que les migrants retenus pour l'étude sont plus à même de vouloir rester en France une fois à la retraite, compte tenu du temps plus important qu'ils vont avoir passé en moyenne dans ce pays.

En second lieu, certaines variables explicatives prises en compte dans les régressions, notamment pour identifier les attaches que les immigrés entretiennent avec leur pays d'accueil et d'origine, sont susceptibles d'engendrer des biais d'endogénéité. A titre d'illustration, un immigré a d'autant moins intérêt à demander la nationalité française et d'autant plus à envoyer de l'argent au pays qu'il entend y retourner. Toutefois, l'enquête ne fournit pas de données suffisamment détaillées sur les motivations du projet de retour ou bien encore sur l'acquisition de la nationalité française pour pouvoir tenir corriger les biais sous-jacents. Seul un suivi longitudinal et durable des populations immigrées spécifiant les motivations des comportements recensés permettrait de mieux comprendre leurs décisions de retour et les conséquences qui en résultent, tant sur le pays d'accueil que sur les pays d'origine. 


\section{Références}

Ahlburg D., Brown R., 1998. Migrants' intention to return home and capital transfers: A study of Togans and Samoans in Australia, Journal of Development Studies, 35, 121-151.

Attias-Donfut C., Wolff F.C., 2005. Transmigration et choix de vie à la retraite, Retraite et Société, 44, 80-107.

Bauer T. K., Sinning M., 2008. An extension of the Blinder-Oaxaca decomposition to nonlinear models. Advances in Statistical Analysis, 92, 197-206.

Bhaumik S., Gang I.N., Yun M.S., 2006. Ethnic conflict and economic disparity: Serbians and Albanians in Kosovo, Journal of Comparative Economics, 34, 754773.

Bohning W., 1981. Estimating the propensity tu guestworkers to leave, Monthly Labor Review, 104, 37-40.

Borjas G., Bratsberg B., 1996. Who leaves? The outmigration of the foreign born, Review of Economics and Statistics, 78, 165-178.

Constant A., Massey D., 2003. Self-selection, earnings, and out-migration: a longitudinal study of immigrants to Germany, Journal of Population Economics, 16, 631-653.

De Coulon A., Wolff F., 2006. The location of immigrants at retirement :Stay/Return or « Va-et-Vient?, IZA Discussion Paper, 2224.

Djajic S., 1989. Migrants in a guest-worker system, Journal of Development Economics, 31, 327-339.

Djajic S ., Milbourne R., 1988. A general equilibrium model of guest-worker migration: A source-country perspective, Journal of International Economics, 25, 335-351.

Domingues Dos Santos M., 2005. Travailleurs maghrébins et portugais en France : le poids de l'origine, Revue Economique, 56, 447-464.

Domingues Dos Santos M., 2006. Attraction des élites et exode des cerveaux : les enjeux économiques d'une concertation entre pays d'origine et pays d'accueil, Horizons Stratégiques, 1, 18-27.

Domingues Dos Santos M., Postel-Vinay F., 2003. Migration as a source of growth : the perspective of a developing country, Journal of Population Economics, 16, 161-175.

Domingues Dos Santos M., Postel-Vinay F., 2005. The impact of temporary migration on human capital accumulation and economic development, Brussels Economic Review.

Duleep H., 1994. Social security and emigration of immigrants, Social Security Bulletin, 57, 37-52.

Dustmann C., 1996. Return migration : the European experience, Economic Policy, 22, 215-250.

Dustmann C., 1997. Differences in the Labour Market Behaviour between Temporary and Permanent Migrant Women, Labour Economics, 4, 29-46.

Dustmann C., 1999. Temporary migration, human capital and language fluency of migrants, Scandinavian Journal of Economics, 1001, (297-314. 
Dustmann C., 2001. Why go back? Return motives of migrants workers, in S. Djajic (ed), International Migration: Trends, Policy, and Economic Impacts, Chapter 11, Routledge.

Dustmann C., 2003. Children and return migration, Journal of Population Economics, $16,815-830$.

Dustmann C., Kirchkamp O., 2002. The optimal migration duration and activity choice after re-migration Journal of Development Economics, 67, 351-372.

Even W.E., Macpherson D.A., 1993. The decline of private-sector unionism and the gender wage gap, Journal of Human Resources, 28, 279-296.

Fairlie R.W., 1999. The absence of the African-American owned business: An analysis of the dynamics of self-employment, Journal of Labor Economics, 17, 80-108.

Fairlie R.W., 2005. An extension of the Blinder-Oaxaca decomposition technique to logit and probit models, Journal of Economic and Social Measurement, 30, 305-316.

Fougère D., Safi M., 2005. L'acquisition de la nationalité française : quels effets sur l'accès à l'emploi des immigrés ?, France Portrait Social, 163-184.

Galor O., Stark O., 1990. Migrants' savings, the probability of return migration, and migrants' performance, International Economic Review, 31, 463-467.

Galor O., Stark O., 1991. The probability of return migration, migrants' effort and migrants' performance, Journal of Development Economics, 35, 399-405.

Glytsos N., 1997. Remitting behaviour of temporary and permanent migrants: the case of Greeks in Germany and Australia, Labour, 11, 409-435.

Jasso G., Rosenzweig M., 1982. Estimating the emigration rates of legal immigrants using administrative and survey data: The 1971 cohort of immigrants to the United States, Demography, 19, 279-290.

Jellal M., Wolff F.C., 2002. Cultural evolutionary altruism: Theory and evidence, European Journal of Political Economy, 18, 241-262.

Jones F.L., 1983. On decomposing the wage gap: A critical comment on Blinder's method, Journal of Human Resources, 18, 126-130.

Mesnard A., 2004. Temporary migration and capital market imperfections, Oxford Economic Papers, 56, 242-262.

Neumark D., 1988, Employer's discriminatory behavior and the estimation of wage discrimination, Journal of Human Resources, 279-295.

Nielsen H.S., 1998. Discrimination and detailed decomposition in a Logit model, Economics Letters, 61, 115-120.

Oaxaca R., Ransom M., 1994. On discrimination and the decomposition of wage differentials, Journal of Econometrics, 61, 5-21.

Ramos, 1992. Out-migration and return migration of Puerto Ricans, Immigration and the workforce, University of Chicago Press, 49-66.

Reagan P., Olsen R., 2000. You can go home again: Evidence from longitudinal data, Demography, 37, 339-350.

Reimers C., 1983. Labor market discrimination against Hispanic and Black men, Review of Economics and Statistics, 65, 570-579. 
Rooth D., Saarela J., 2007. Selection in migration and return migration: Evidence from micro data, Economics Letters, 94, 90-95.

Sloan F.A., Zhang H.H., Wang J., 2002. Upstream intergenerational transfers, Southern Economic Journal, 69, 363-380.

Stark, 1992. The migration of labor, Backwell, Oxford.

Steiner V., Velling J., 1994. Re-migration behavior and expected duration of stay of guest workers in Germany, The economic consequences of immigration to Germany, Physical-Verlag, 101-119.

Yun M.-S., 2004. Decomposing differences in the first moment, Economics Letters, $82,275-280$. 
Tableau 1. Description des données

\begin{tabular}{|c|c|c|c|c|c|}
\hline \multirow{2}{*}{\multicolumn{2}{|c|}{ Variables }} & \multicolumn{3}{|c|}{ Pays d'origine du migrant } & \multirow{2}{*}{ Ensemble } \\
\hline & & Italie & Espagne & Portugal & \\
\hline \multicolumn{6}{|c|}{ Variable dépendante } \\
\hline \multicolumn{2}{|c|}{$\begin{array}{l}\text { Intention de retour } \\
\text { Caractéristiques démo- } \\
\text { économiques }\end{array}$} & 0,131 & 0,266 & 0,532 & 0,371 \\
\hline \multicolumn{2}{|c|}{ Sexe féminin } & 0,455 & 0,537 & 0,465 & 0,480 \\
\hline \multicolumn{2}{|l|}{ Age } & 53,838 & 53,638 & 52,947 & 53,328 \\
\hline \multicolumn{2}{|c|}{ Vit en couple } & 0,888 & 0,847 & 0,896 & 0,882 \\
\hline \multicolumn{2}{|c|}{ Nombre d'années d'éducation } & 9,508 & 8,528 & 6,391 & 7,657 \\
\hline Revenu du & Moyenne & 9,971 & 9,825 & 9,863 & 9,880 \\
\hline \multirow[t]{3}{*}{ ménage (log) } & Quartile 1 & 6,634 & 9,496 & 9,617 & 9,604 \\
\hline & Quartile 2 & 10,043 & 9,880 & 9,992 & 9,987 \\
\hline & Quartile 3 & 10,500 & 10,327 & 10,309 & 10,331 \\
\hline \multirow[t]{7}{*}{ Région } & Région parisienne & 0,154 & 0,254 & 0,510 & 0,361 \\
\hline & Bassin parisien & 0,039 & 0,040 & 0,114 & 0,078 \\
\hline & Nord & 0,078 & 0,023 & 0,040 & 0,045 \\
\hline & Est & 0,240 & 0,037 & 0,062 & 0,100 \\
\hline & Sud-Ouest & 0,059 & 0,212 & 0,123 & 0,129 \\
\hline & Centre-Est & 0,224 & 0,105 & 0,117 & 0,140 \\
\hline & Méditerranée & 0,207 & 0,331 & 0,034 & 0,148 \\
\hline \multicolumn{6}{|c|}{ Caractéristiques migratoires } \\
\hline \multicolumn{2}{|c|}{ Age à la migration } & 12,480 & 15,489 & 20,855 & 17,544 \\
\hline \multicolumn{2}{|c|}{ Durée de la migration } & 41,358 & 38,150 & 32,091 & 35,784 \\
\hline \multirow[t]{5}{*}{ Arrivée } & Avant 1960 & 0,567 & 0,268 & 0,014 & 0,209 \\
\hline & $1960-1964$ & 0,165 & 0,316 & 0,115 & 0,175 \\
\hline & $1965-1969$ & 0,095 & 0,223 & 0,364 & 0,265 \\
\hline & $1970-1974$ & 0,064 & 0,102 & 0,374 & 0,234 \\
\hline & Après 1975 & 0,109 & 0,090 & 0,133 & 0,117 \\
\hline \multirow{2}{*}{\multicolumn{2}{|c|}{$\begin{array}{l}\text { Difficulté à parler le français } \\
\text { Attaches avec la France }\end{array}$}} & 0,140 & 0,274 & 0,433 & 0,324 \\
\hline & & & & & \\
\hline \multicolumn{2}{|c|}{ Conjoint né en France } & 0,550 & 0,446 & 0,142 & 0,314 \\
\hline \multicolumn{2}{|c|}{ Enfants vivant en France } & 0,908 & 0,924 & 0,926 & 0,921 \\
\hline \multicolumn{2}{|c|}{ Nationalité française } & 0,497 & 0,506 & 0,175 & 0,332 \\
\hline \multicolumn{2}{|c|}{ Propriétaire } & 0,765 & 0,757 & 0,580 & 0,667 \\
\hline \multirow{2}{*}{\multicolumn{2}{|c|}{$\begin{array}{l}\text { Sentiment de discrimination } \\
\text { Attaches avec le pays d'origine }\end{array}$}} & 0,221 & 0,169 & 0,121 & 0,157 \\
\hline & & & & & \\
\hline \multicolumn{2}{|c|}{ Parents au pays d'origine } & 0,196 & 0,266 & 0,400 & 0,318 \\
\hline \multicolumn{2}{|c|}{ Enfants vivant au pays } & 0,078 & 0,107 & 0,119 & 0,106 \\
\hline \multicolumn{2}{|c|}{ Autre famille au pays } & 0,930 & 0,963 & 0,965 & 0,956 \\
\hline \multicolumn{2}{|c|}{ Envoi d'argent au pays } & 0,081 & 0,153 & 0,456 & 0,293 \\
\hline Nombre & d'observations & 358 & 354 & 767 & 1479 \\
\hline
\end{tabular}

Source: Enquête PRI 2003. 
Tableau 2. Les déterminants des intentions de retour des migrants

\begin{tabular}{|c|c|c|c|c|c|c|}
\hline Variables & & & & & & \\
\hline & Effet & t-test & Effet & t-test & Effet & t-test \\
\hline Var. démo-économiques & & & & & marginal & \\
\hline Sexe féminin & $-3,9 \%$ & $-1,48$ & $-5,7 \%{ }^{* *}$ & $-2,10$ & $-6,3 \%{ }^{* *}$ & $-2,20$ \\
\hline Age & $-0,6 \%{ }^{* *}$ & $-2,22$ & & & & \\
\hline Vit en couple & $2,4 \%$ & 0,56 & $2,9 \%$ & 0,68 & $5,5 \%$ & 1,20 \\
\hline Nombre d'années d'éducation & $-1,6 \%{ }^{* * *}$ & $-3,97$ & $-1,0 \%{ }^{* *}$ & $-2,51$ & $-0,6 \%$ & $-1,31$ \\
\hline Revenu du ménage (log) & $3,1 \%{ }^{*}$ & 1,77 & $3,1 \%{ }^{*}$ & 1,73 & $3,4 \%{ }^{* *}$ & 1,79 \\
\hline Vit en région parisienne & $20,0 \%{ }^{* * *}$ & 7,06 & $19,8 \%^{* * *}$ & 6,87 & $13,8 \%^{* * *}$ & 4,49 \\
\hline Migration & & & & & & \\
\hline Age à la migration & & & $-0,2 \%$ & $-0,68$ & $-0,8 \%{ }^{* * *}$ & $-2,77$ \\
\hline Durée de migration & & & $-1,9 \%{ }^{* * *}$ & $-6,20$ & $-1,6 \%{ }^{* * *}$ & $-5,01$ \\
\hline Difficulté à parler le Français & & & $10,4 \%{ }^{* * *}$ & 3,35 & $8,4 \%{ }^{* * *}$ & 2,60 \\
\hline Attaches avec la France & & & & & & \\
\hline Conjoint né en France & & & & & $-13,9 \%{ }^{* * *}$ & $-3,77$ \\
\hline Enfants vivant en France & & & & & $-3,5 \%$ & $-0,63$ \\
\hline Nationalité française & & & & & $-12,9 \%{ }^{* * *}$ & $-3,75$ \\
\hline Propriétaire & & & & & $-11,1 \%{ }^{* * *}$ & $-3,59$ \\
\hline Sentiment de discrimination & & & & & & \\
\hline Attaches avec le pays d'origine & & & & & $9,1 \%^{* *}$ & 2,24 \\
\hline Parents vivant au pays & & & & & $7,3 \%^{* *}$ & 2,38 \\
\hline Enfants vivant au pays & & & & & $15,9 \%{ }^{* * *}$ & 3,26 \\
\hline Autre famille au pays & & & & & $0,1 \%$ & 0,01 \\
\hline Envoi d'argent au pays & & & & & $21,6 \%{ }^{* * *}$ & 6,51 \\
\hline Pays de naissance & & & & & & \\
\hline Origine portugaise & $24,3 \%{ }^{* * *}$ & 8,41 & $14,0 \%{ }^{* * *}$ & 4,53 & $5,8 \%{ }^{*}$ & 1,69 \\
\hline Probabilité moyenne estimée (en \%) & & & & & & \\
\hline Nombre d'observations & & & & & & \\
\hline Pseudo $\mathrm{R}^{2}$ & & & & & & \\
\hline Log vraisemblance & & & & & & \\
\hline
\end{tabular}

Source: Enquête PRI 2003.

Note: effets marginaux calculés à partir de Modèles Probit. Les seuils de significativité retenus sont respectivement de $1 \%$ $\left(^{* * *}\right), 5 \%\left(^{* *}\right)$ et $10 \%\left(\left(^{*}\right)\right.$. 
Tableau 3. Décomposition détaillée des intentions de retour des migrants

\begin{tabular}{|c|c|c|c|}
\hline Décomposition & $\begin{array}{c}(1) \\
\beta^{*}=\beta_{A}\end{array}$ & $\begin{array}{c}(2) \\
\beta^{*}=\beta_{B}\end{array}$ & $\begin{array}{c}(3) \\
\beta^{*}=\beta\end{array}$ \\
\hline \multirow[t]{2}{*}{ Différence totale } & 0,331 & 0,331 & 0,331 \\
\hline & $100 \%$ & $100 \%$ & $100 \%$ \\
\hline \multirow[t]{2}{*}{ Différence de caractéristiques } & 0,211 & 0,228 & 0,245 \\
\hline & $63,7 \%$ & $69,0 \%$ & $74,1 \%$ \\
\hline \multirow[t]{2}{*}{ Différences de coefficients } & 0,120 & 0,103 & 0,086 \\
\hline & $36,3 \%$ & $31,0 \%$ & $25,9 \%$ \\
\hline \multicolumn{4}{|l|}{$\begin{array}{l}\text { Différence de caractéristiques: effets } \\
\text { détaillés }\end{array}$} \\
\hline \multirow[t]{2}{*}{ Sexe féminin } & $\begin{array}{c}0,001 \\
(0,001)\end{array}$ & $\begin{array}{c}0,002 \\
(0,001)\end{array}$ & $\begin{array}{c}0,002 \\
(0,001)\end{array}$ \\
\hline & $0,6 \%$ & $0,9 \%$ & $0,8 \%$ \\
\hline \multirow[t]{2}{*}{ Vit en couple } & $\begin{array}{c}0,000 \\
(0,002)\end{array}$ & $\begin{array}{c}0,002 \\
(0,002)\end{array}$ & $\begin{array}{c}0,001 \\
(0,002)\end{array}$ \\
\hline & $0,1 \%$ & $1,0 \%$ & $0,6 \%$ \\
\hline \multirow[t]{2}{*}{ Nombre d'années d'éducation } & $\begin{array}{c}0,011 \\
(0,010)\end{array}$ & $\begin{array}{c}0,038 \\
(0,014)\end{array}$ & $\begin{array}{c}0,032 \\
(0,008)\end{array}$ \\
\hline & $5,2 \%$ & $16,6 \%$ & $13,1 \%$ \\
\hline \multirow[t]{2}{*}{ Revenu du ménage (log) } & $\begin{array}{c}0,000 \\
(0,000)\end{array}$ & $\begin{array}{c}0,000 \\
(0,000)\end{array}$ & $\begin{array}{c}0,000 \\
(0,000)\end{array}$ \\
\hline & $0,1 \%$ & $-0,1 \%$ & $0,0 \%$ \\
\hline \multirow[t]{2}{*}{ Vit en région parisienne } & $\begin{array}{c}0,080 \\
(0,015)\end{array}$ & $\begin{array}{c}0,043 \\
(0,010)\end{array}$ & $\begin{array}{c}0,066 \\
(0,008)\end{array}$ \\
\hline & $38,0 \%$ & $18,6 \%$ & $26,8 \%$ \\
\hline \multirow[t]{2}{*}{ Age à la migration } & $\begin{array}{c}-0,023 \\
(0,013)\end{array}$ & $\begin{array}{c}0,009 \\
(0,022)\end{array}$ & $\begin{array}{c}-0,014 \\
(0,011)\end{array}$ \\
\hline & $-11,1 \%$ & $3,9 \%$ & $-5,6 \%$ \\
\hline \multirow[t]{2}{*}{ Durée de la migration } & 0,106 & 0,118 & 0,132 \\
\hline & $\begin{array}{c}(0,018) \\
50,4 \%\end{array}$ & $\begin{array}{c}(0,026) \\
51,9 \%\end{array}$ & $\begin{array}{c}(0,014) \\
53,8 \%\end{array}$ \\
\hline \multirow[t]{3}{*}{ Difficulté à parler le Français } & 0,035 & 0,016 & 0,026 \\
\hline & $(0,012)$ & $(0,009)$ & $(0,007)$ \\
\hline & $16,8 \%$ & $7,2 \%$ & $10,5 \%$ \\
\hline
\end{tabular}

Source: Enquête PRI 2003.

Note : les résultats reportés sont obtenues à partir d'une décomposition non-linéaire des intentions de retour, les écarts-type indiqués entre parenthèses sous les coefficients étant obtenus à partir d'une technique de bootstrap. 
Tableau 4. Projets de vie à l'époque d'arrivée en France

\begin{tabular}{|l|c|c|c|}
\hline \multirow{2}{*}{ Réponses } & \multicolumn{2}{|c|}{ Pays d'origine du migrant } & \multirow{2}{*}{ Ensemble } \\
\cline { 2 - 3 } & Espagne ou Italie & Portugal & \\
\hline Passer la vie professionnelle en & 5,9 & 17,6 & 13,6 \\
$\begin{array}{l}\text { France, puis rentrer au pays } \\
\text { Rester quelque temps en France, }\end{array}$ & 28,9 & 35,9 & 33,5 \\
puis rentrer au pays & & & \\
Rester quelque temps en France, & 3,6 & 1,0 & 1,9 \\
puis aller dans un autre pays & 34,2 & 21,4 & 25,7 \\
Rester définitivement en France & 27,5 & 24,1 & 25,2 \\
Sans projet précis, autre & 100,0 & 100,0 & 100,0 \\
\hline Total (en \%) & &
\end{tabular}

Source: Enquête PRI 2003. 